\title{
SAÚDE DO HOMEM NA BAHIA: A INTERNAÇÃO HOSPITALAR DE ADULTOS NOS ANOS 2000 E 2010
}

\author{
MEN'S HEALTH IN BAHIA: HOSPITALIZATION \\ OF ADULT SUBJECTS BETWEEN 2000 AND 2010
}

\section{Renato Queiroz dos Santos Júnior ${ }^{1}$, Augusto Cesar Costa Cardoso ${ }^{2}$, Suzana Costa Carvalho ${ }^{3}$,}

\author{
Zenaide Calazans de Oliveira ${ }^{4}$, Márcia de Paulo Costa Mazzei $^{5}$
'Mestre em Saúde Coletiva. Professor na Universidade Estadual de Feira de Santana e Técnico da Secretaria Estadual da Saúde, \\ Superintendência de Vigilância à Saúde. Feira de Santana, Bahia, Brasil. renatoqueiroz@superig.com.br \\ ${ }^{2}$ Doutor em Saúde Coletiva. Professor da Universidade do Estado da Bahia e da Escola Bahiana de Medicina e Saúde Pública. \\ Salvador, Bahia, Brasil. cesarcardoso11@gmail.com \\ ${ }^{3}$ Técnica da Secretaria Estadual da Saúde, Superintendência de Recursos Humanos. Salvador, Bahia, Brasil. suzanaccarvalho@gmail.com \\ ${ }^{4}$ Técnica da Secretaria Estadual da Saúde, Superintendência de Vigilância à Saúde. Salvador, Bahia, Brasil. zenaide.oliveira@saude.ba.gov.br \\ ${ }^{5}$ Técnica da Secretaria Estadual da Saúde, Superintendência de Vigilância à Saúde. Salvador, Bahia, Brasil. marcia.mazzei@saude.ba.gov.br
}

RESUMO | Descrever a situação de saúde dos homens no Estado da Bahia, na faixa etária de 20 a 59 anos, considerando as internações hospitalares nos anos de 2000 e 2010. Método: Estudo epidemiológico transversal, descritivo, série temporal com dados secundários e retrospectivos de internação hospitalar de homens no estado da Bahia no período de 2000 a 2010. Os dados utilizados foram extraídos do SIH/SUS e os sobre a população são do IBGE. Resultados: Este estudo pretende apontar os principais problemas de saúde da população masculina a serem enfrentados pelos gestores e usuários nos diversos níveis do SUS. Observa-se na faixa etária de 20 a 59 anos, na população masculina, o predomínio acentuado de internações em 2010 por causas externas, na Bahia, na rede hospitalar do SUS, no comparativo com o ano de 2000, embora as variações das doenças infectoparasitárias e neoplasias, no mesmo período, sejam mais elevadas que as causas externas. Há também crescimento das internações hospitalares por doenças infectoparasitárias, transtornos mentais e doenças osteomusculares. Conclusões: A menor frequência dos homens aos serviços de atenção primária faz com que esse grupo populacional fique privado da proteção adequada à preservação de sua saúde, propiciando o uso de procedimentos desnecessários que poderiam ser evitados, caso a procura pela atenção houvesse ocorrido em momento oportuno. Há necessidade de serem criadas redes de atendimento aos homens, além da qualificação dos profissionais de saúde da atenção básica e uma maior sensibilização por parte dos gestores Estaduais e Municipais.

Palavras-chave: Saúde do homem. Taxa de internações hospitalares. Saúde Pública.

\begin{abstract}
The aim of this study was to describe the health status of men in the State of Bahia, aged 20 to 59 years, considering hospital admissions in 2000 and 2010. Method: Cross-sectional epidemiological study, descriptive, time series with secondary data and retrospective hospital admission of men in the state of Bahia from 2000 to 2010 . The data used were extracted from the SIH / SUS and the data about the population are from IBGE. Results: This study intends to point out the main health problems of male population to be faced by managers and users in different levels of SUS program. Higher hospitalizations rates due to external causes in Bahia in SUS hospital network in 2010 was observed in male population aged 20 to 59 years old than in 2000 , even though variations in infectious and parasitic diseases and neoplasms are more prevalent than external causes in same population. There was also an increase in hospital admissions for infectious diseases, mental disorders and musculoskeletal diseases. Conclusions: Lower frequency of men to primary care services makes this population group deprived of adequate protection to preserve their health, providing unnecessary procedures that could be avoided if the search for attention had occurred in a timely manner. There is a need to create care networks for men, as well as to qualify health professionals in basic care and a greater awareness by State and Municipal managers to this issue.
\end{abstract}

Keywords: Men's Health. Rate of hospital admissions. Public health 


\section{INTRODUÇÃO}

No Brasil, a política de saúde do homem vem sendo construída nos últimos anos levando-se em conta as singularidades do grupo, além da ênfase na melhoria do acesso e satisfação deste público, historicamente alijado das políticas e cuidados, em todos os níveis de atenção à saúde'. Nesse sentido, a Política Nacional de Atenção Integral à Saúde do Homem (PNAISH) traduz um longo anseio da sociedade ao reconhecer que os agravos do sexo masculino são relevantes problemas de interesse da saúde pública.

A PNAISH está alinhada à Atenção Primária à Saúde, porta de entrada preferencial do Sistema Único de Saúde - SUS, onde a demanda por serviços de saúde é dominantemente voltada às mulheres, aponta que os homens não são considerados protagonistas de seus cuidados ${ }^{2}$. Além disso, os estudos sobre morbidade e mortalidade referentes à população masculina ainda são incipientes.

Não existem paralelos entre o enfoque de saúde das mulheres e dos homens, nem semelhanças na maneira de interatuar com $\circ$ sistema de atenção à saúde. Em geral, os homens são pouco cuidadosos com sua saúde e, em suas interações com os serviços de saúde, muitos assumem uma fantasia de "carvalho maciço"3. Outros autores relatam, ainda, que os homens associam o cuidar ao âmbito feminino e por se sentirem inatingíveis, se expõem mais, ficando vulneráveis ${ }^{4}$. A ausência dos homens ou sua invisibilidade, nesses serviços, é uma característica da identidade masculina relacionada ao seu processo de socialização, agregada à desvalorização do autocuidado e à preocupação incipiente com a saúde.

Além disso, é bastante disseminada a ideia de que as unidades básicas de saúde (UBS) são serviços destinados quase que exclusivamente para mulheres, crianças e idosos 5 . De fato, os serviços de saúde são frequentados, principalmente, por mulheres e composto por uma equipe de profissionais formada, em sua maioria, também por mulheres.

Assim, admitem-se as dificuldades de acesso da população masculina aos serviços, uma vez que, habitualmente, a mesma não se reconhece como alvo de atendimento dos programas de saúde. Neste contexto, os homens preferem utilizar outros serviços de saúde, como farmácias ou prontossocorros, que responderiam mais objetivamente às suas demandas. Nesses lugares, os homens seriam atendidos mais rapidamente e conseguiriam expor seus problemas com mais facilidade ${ }^{5}$.

Desta forma, a população masculina interatua com - sistema de saúde de forma episódica e os serviços de atenção primária apresentam dificuldades para acionar práticas de prevenção, proteção e promoção da saúde, resultando na inadequada condição de saúde masculina ${ }^{3,6}$, o que tem como consequência - agravo das morbidades pelo retardamento na atenção e maior custo para o sistema de saúde, uma vez que o adentramento ao sistema de saúde ocorre principalmente pela assistência ambulatorial e hospitalar ${ }^{5}$.

O objetivo da pesquisa é descrever a situação de saúde dos homens no Estado da Bahia, na faixa etária de 20 a 59 anos, considerando as internações hospitalares nos anos de 2000 e 2010.

\section{MATERIAL E MÉTODO}

Este trabalho é um estudo epidemiológico transversal, descritivo, série temporal com dados secundários e retrospectivos da situação de saúde dos homens no Estado da Bahia, na faixa etária de 20 a 59 anos, considerando as internações hospitalares para este grupo, nos anos 2000 e 2010.

Os dados utilizados neste estudo referentes às internações hospitalares foram extraídos do Sistema de Informações Hospitalares do SUS (SIH/SUS) e os sobre a população são do Instituto Brasileiro de Geografia e Estatística (IBGE). O SIH/SUS é gerido pelo Ministério da Saúde, através da Secretaria de Assistência à Saúde, em conjunto com as Secretarias Estaduais de Saúde e as Secretarias Municipais de Saúde, sendo processado pelo Datasus Departamento de Informática do SUS, que possui dados sobre as internações realizadas em todos os 
hospitais vinculados ao SUS no país, desde 1991, excluindo, portanto, as internações custeadas diretamente ou cobertas por seguro-saúde.

Estima-se que o SIH/SUS cobre cerca de 60 a $70 \%$ das internações hospitalares realizadas no país, variando de acordo com a região. É importante mencionar, ainda, que eventuais reinternações e transferências de um mesmo paciente para outros hospitais não são identificadas, o que pode resultar em uma contagem cumulativa. $O$ documento básico do SIH/SUS é a Autorização de Internação Hospitalar (AIH), que é preenchida no hospital, habilita a internação do paciente e gera valores para o pagamento da internação ${ }^{7}$.

Optou-se por analisar os dados do Brasil e do estado da Bahia, inclusive, de acordo com suas Macrorregiões de Saúde, de acordo com Plano Diretor de Regionalização (PDR) a Bahia estava subdividida em nove Macrorregiões de Saúde, a saber: Centro-Leste, Centro-Norte, Extremo Sul, Leste, Nordeste, Norte, Oeste, Sudoeste e Sul8. A partir de 2014, as macrorregiões de saúde do estado da Bahia passaram a corresponder aos Núcleos Regionais de Saúde (NRS), conforme Lei Estadual $n^{\circ}$ 13.204/20149. Dessa forma, as informações foram desagregadas e apresentadas para os nove NRS do Estado - Norte, Sul, Leste, Oeste, Nordeste, Sudoeste, Centro Leste, Centro Norte, Extremo Sul - conforme o Plano Diretor de Regionalização (PDR). As populações residentes por sexo e região geográfica, denominadores no cálculo das taxas de internação, são informações dos Censos 2000 e 2010 do IBGE por sexo e idade para os anos 2000 e 2010.

Foram utilizados, principalmente, cálculos dos coeficientes e proporções de internações dos homens, e comparados os indicadores no tempo e no espaço. A análise da população de estudo também foi realizada por faixa etária (20-29, 30-39, 40-49 e 50-59 anos), a fim de identificar singularidades inerentes aos estágios da vida e graus diferentes de acesso ao sistema de saúde para internação hospitalar. Os recortes adotados, em grupos de causas, principais agravos e doenças, e a também distribuição por NRS visam subsidiar a reorientação de ações voltadas às necessidades da população masculina na Bahia e desta forma, ampliar o acesso e a resolutividade das práticas assistenciais no SUS para este grupo específico. Estudar a singularidade do processo saúde-doença entre segmentos masculinos permite a elaboração de políticas e ações de saúde mais integrais.

Não houve a necessidade de aprovação desta pesquisa pelo Comitê de Ética e Pesquisa por se tratar de dados secundários e públicos, conforme a Resolução 466/1 2 do Conselho Nacional de Saúde.

\section{RESULTADOS}

O valor total gasto com internações hospitalares em homens na faixa etária de 20 a 59 anos de idade no Sistema Único de Saúde em 2010 no Estado da Bahia foi $R \$ 110.093 .299,79$, que representou $18,8 \%$ do total gasto com internações hospitalares.

Os grupos de causas de hospitalização mais frequentes na referida população de estudo naquele ano foram, respectivamente: causas externas, doenças do aparelho digestivo, infecto parasitárias, do aparelho circulatório e do respiratório. Para as mulheres, o grupo de causas mais frequente foi o das doenças do aparelho geniturinário. No sexo feminino, em 2010, as neoplasias apareceram como o quarto grupo de causas de internação, enquanto para os homens são a oitava, mas esse grupo de causas foi o que apresentou o maior aumento percentual $(34,8 \%)$ das taxas de internação no sexo masculino entre 2000 e 2010 (Tabela 1).

Tabela 1. Taxa* de internação hospitalar por 10.000 habitantes e variação percentual**, segundo grandes grupos de causas. Bahia, 2000 e 2010.

\begin{tabular}{lcccccc}
\hline \multirow{2}{*}{ Grandes grupos de causas } & \multicolumn{3}{c}{ Homens } & \multicolumn{3}{c}{ Mulheres } \\
\cline { 2 - 7 } & 2000 & 2010 & $\begin{array}{c}\text { Variação } \\
\text { percentual }\end{array}$ & 2000 & 2010 & $\begin{array}{c}\text { Variação } \\
\text { percentual }\end{array}$ \\
\hline Causas externas & 58,9 & 70,7 & 20,0 & 21,4 & 19,4 & $-9,4$ \\
D. do Ap. Digestivo & 70,8 & 54,8 & $-22,6$ & 81,9 & 61,4 & $-25,1$ \\
D. Infectoparasitárias & 40,1 & 49,9 & 24,4 & 42,7 & 50,9 & 19,2
\end{tabular}


Tabela 1. Taxa* de internação hospitalar por 10.000 habitantes e variação percentual**, segundo grandes grupos de causas. Bahia, 2000 e 2010.

\begin{tabular}{|c|c|c|c|c|c|c|}
\hline \multirow[b]{2}{*}{ Grandes grupos de causas } & \multicolumn{3}{|c|}{ Homens } & \multicolumn{3}{|c|}{ Mulheres } \\
\hline & 2000 & 2010 & $\begin{array}{l}\text { Variação } \\
\text { percentual }\end{array}$ & 2000 & 2010 & $\begin{array}{l}\text { Variação } \\
\text { percentual }\end{array}$ \\
\hline D. do Ap. Circulatório & 44,8 & 35,8 & $-20,0$ & 62,5 & 40,4 & $-35,4$ \\
\hline D. do Ap. Respiratório & 71,2 & 35,1 & $-50,8$ & 95,8 & 40,7 & $-57,5$ \\
\hline D. do Ap. Geniturinário & 28,5 & 22,4 & $-21,5$ & $\begin{array}{c}189 \\
4\end{array}$ & 77.2 & $-59,2$ \\
\hline Transtornos Mentais & 32,6 & 13,0 & $-60,1$ & 38,9 & 11,5 & $-70,4$ \\
\hline Neoplasia & 10,2 & 13,8 & 34,8 & 54 & 45,5 & $-15,8$ \\
\hline D. do Sistema & & & & & & \\
\hline Osteomuscular & 18,1 & 13,2 & $-27,2$ & 17,1 & 7,8 & $-54,4$ \\
\hline
\end{tabular}

No comparativo entre 2000 e 2010 , observou-se redução nas taxas de internações hospitalares na maioria dos grandes grupos de causa na Bahia e no Brasil, tanto para homens quanto para mulheres (Tabelas 1 e 2). Os grupos de causa que apresentaram aumento nas taxas de internação para o sexo masculino na Bahia e no Brasil foram: neoplasias, doenças infectoparasitárias e causas externas (Tabelas 1 e 2).

$\mathrm{Na}$ Bahia, as taxas de internação hospitalar no grupo feminino são superiores às no grupo masculino, em 2000 e 2010, exceto para os grupos de internações por causas externas, transtornos mentais e doenças osteomusculares. Essa situação corrobora com estudos sobre diferenças de gênero na saúde que apontam que embora vivam mais do que os homens, as mulheres relatam mais morbidade e utilizam mais os serviços de saúde em todos os níveis de atenção.

Tabela 2. Taxa* de internação hospitalar por 10.000 habitantes e variação percentual**, segundo grandes grupos de causas. Brasil, 2000 e 2010.

\begin{tabular}{lcccccc}
\hline \multirow{2}{*}{ Grandes grupos de causas } & \multicolumn{3}{c}{ Homens } & \multicolumn{3}{c}{ Mulheres } \\
\cline { 2 - 7 } & 2000 & 2010 & $\begin{array}{c}\text { Variação } \\
\text { percentual }\end{array}$ & 2000 & 2010 & $\begin{array}{c}\text { Variação } \\
\text { percentual }\end{array}$ \\
\hline Causas externas & 67,4 & 81,9 & 21,5 & 20,9 & 24,1 & 15,3 \\
D. do Ap. Digestivo & 67,5 & 56,2 & $-16,7$ & 62,1 & 55,5 & $-10,6$ \\
D. Infectoparasitárias & 34,9 & 35,1 & 0,6 & 28,3 & 31,3 & 10,6 \\
D. do Ap. Circulatório & 50,8 & 44,9 & $-11,6$ & 60,7 & 45,8 & $-24,5$ \\
D. do Ap. Respiratório & 54,7 & 33,3 & $-39,1$ & 59,0 & 31,2 & $-47,1$ \\
D. do Ap. Geniturinário & 26,0 & 22,8 & $-12,3$ & 98,9 & 64,2 & $-35,1$ \\
Transtornos Mentais & 55,9 & 31,1 & $-44,4$ & 28,1 & 15,7 & $-44,1$ \\
Neoplasia & 13,9 & 19,4 & 39,6 & 39,9 & 44,2 & 10,8 \\
D. do Sistema & 19,4 & 14,6 & $-24,7$ & 14,0 & 9,2 & $-34,3$ \\
Osteomuscular & & &
\end{tabular}

Fonte: Ministério da Saúde/Datasus-SIH-SUS; Sesab/Suvisa/DIS.

*Taxas de internação calculadas para 10.000 habitantes, para a faixa etária de 20 a 59 anos.

**Variação percentual entre os anos 2000 e 2010.

\section{Internações por causas externas}

As causas externas constituíram o principal grupo de causas de internações da população masculina de 20 a 59 anos na Bahia e no Brasil em 2010. As causas externas de morbidade identificadas neste estudo correspondem aos capíltulos XIX (Lesões, envenenamento e algumas outras conseqüências de causas externas) e XX (Causas externas de morbidade e de mortalidade) da Classificação Internacional de Doenças - CID 10, publicada pela Organização Mundial de Saúde (OMS). Deste modo se caracterizam por: lesões decorrentes de acidentes relacionados ao trânsito, afogamento, envenenamento, quedas ou queimaduras, e as violências, que incluem as agressões, tentativas de suicídio, abusos físicos, sexuais e psicológicos.

Observa-se uma tendência de crescimento na Bahia e no Brasil, entre 2000 e 2010, para as taxas de internação por causas externas nos homens ( 20 a 59 anos), inclusive em todas as faixas etárias desagregadas. 
Destaca-se ainda que as maiores taxas foram identificadas para os adultos jovens, com idade de 20 a 29, tanto na Bahia $(79,5)$ quanto no Brasil $(91,5)$ - Tabela 3.

Tabela 3. Taxas* de internação hospitalar e variação percentual** por Causas Externas, por faixa etária, em homens. Bahia e Brasil, 2000 e 2010.

\begin{tabular}{lcccccc}
\hline Faixa etária & \multicolumn{3}{c}{ Bahia } & \multicolumn{3}{c}{ Brasil } \\
& 2000 & 2010 & Variação \% & 2000 & 2010 & Variação \% \\
\hline 20 a 29 anos & 63,1 & 79,5 & 26,6 & 74,8 & 91,5 & 22,3 \\
30 a 39 anos & 61,3 & 71,8 & 17,1 & 68,0 & 81,6 & 20,0 \\
40 a 49 anos & 51,3 & 62,8 & 22,4 & 61,6 & 76,1 & 23,5 \\
50 a 59 anos & 53,1 & 60,0 & 13,0 & 57,4 & 70,6 & 23,0 \\
\hline
\end{tabular}

Fonte: Ministério da Saúde/Datasus-SIH-SUS; Sesab/Suvisa/DIS.

*Taxas de internação calculadas para 10.000 habitantes, para a faixa etária de 20 a 59 anos.

**Variação percentual entre os anos 2000 e 2010.

Observou-se também o aumento das taxas de internação por causas externas em quase todas os NRS da Bahia, no comparativo dos anos de 2000 e 2010. No NRS Extremo Sul, as taxas de internação de tais agravos mais que dobraram (de 53,3 para 117,5 , crescimento de 120,4\%), em contraste com o NRS Sul, onde houve uma redução de $4,2 \%$ no mesmo período $(82,3$ para 81,8$)$ - Tabela 4.

Tabela 4. Taxas* de internação hospitalar e variação percentual** por causas externas segundo NRS, em homens. Bahia, 2000 e 2010.

Causas externas

\begin{tabular}{lccc}
\hline \multicolumn{1}{c}{ NRS } & \multicolumn{2}{c}{ Ano } & Variação percentual (\%) \\
\hline Extremo Sul & 2000 & 2010 & 120,4 \\
Sul & 53,3 & 117,5 & $-4,2$ \\
Sudoeste & 82,3 & 78,9 & 29,2 \\
Norte & 60,7 & 78,4 & 28,3 \\
Leste & 54,2 & 69,6 & 19,9 \\
Centro-Leste & 57,5 & 68,9 & 12,3 \\
\hline
\end{tabular}

Fonte: Sesab/Suvisa/DIS-SIH-SUS.

*Taxas de internação calculadas para 10.000 habitantes, para a faixa etária de 20 a 59 anos.

**Variação percentual entre os anos 2000 e 2010.

Entre as internações por causas externas nos homens, na faixa etária de 20 a 59 anos, as taxas mais elevadas, em 2000 e 2010 foram, respectivamente, para: Outras causas externas de traumatismos acidentais, Acidentes de transporte e Agressões. Porém, o maior crescimento no período estudado ocorreu na taxa de internação por Eventos cuja intenção é indeterminada (146,2\%).

Tabela 5. Taxas* de internação hospitalar e variação percentual** por causas externas, em homens. Bahia, 2000 e 2010.

\begin{tabular}{lccc}
\hline Causas externas & 2000 & 2010 & $\begin{array}{c}\text { Variação } \\
\text { percentual (\%) }\end{array}$ \\
\hline Outras causas externas de traumatismos acidentais & 30,2 & 42,0 & 39,1 \\
Acidentes de transporte & 7,9 & 11,7 & 48,1 \\
Agressões & 7,2 & 9,1 & 26,4 \\
Eventos cuja intenção é indeterminada & 1,3 & 3,2 & 146,2 \\
Lesões autoprovocadas intencionalmente & 0,8 & 0,6 & $-25,0$ \\
Intervenções legais e operações de guerra & $0,0 * * *$ & $0,0 * * *$ & 0,0 \\
\hline
\end{tabular}

Fonte: Sesab/Suvisa/DIS-SIH-SUS.

*Taxas de internação calculadas para 10.000 habitantes, para a faixa etária de 20 a 59 anos.

**Variação percentual entre os anos 2000 e 2010.

***Valor resultante de arredondamento de um dado originalmente positivo $-0,01$. 
As taxas de internação por acidentes de transporte apresentaram um crescimento de $48,1 \%$ no período de 2000 a 2010 (Tabela 5) e neste último ano 54,0\% das hospitalizações por acidente de transporte foram devido aos acidentes com motociclistas. Destaca-se ainda, que a maioria $(78,4 \%)$ dos acidentes que envolvem motociclistas acontecem entre os homens na faixa etária de 20 a 39 anos (Gráfico 1).

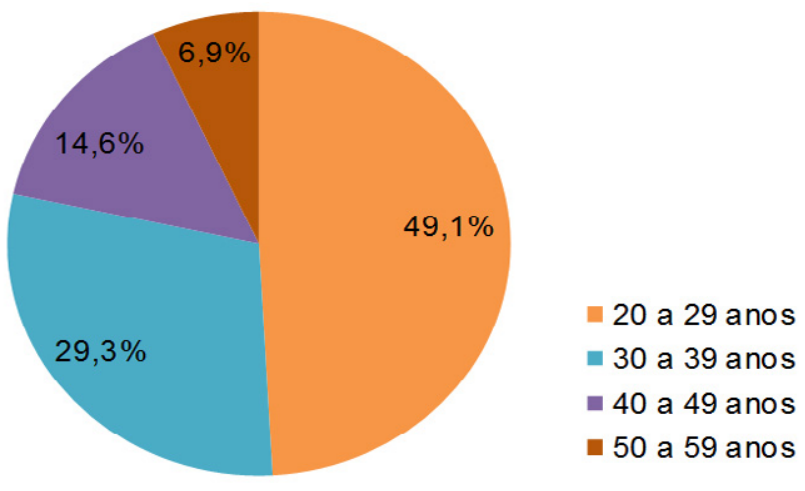

Gráfico 1. Proporção de internações hospitalares* por acidentes de motociclistas, segundo faixa etária. Bahia, 2010.

*Internação hospitalares para homens na faixa etária de 20 a 59 anos. Fonte: Sesab/Suvisa/DIS-SIH-SUS.

As taxas de internação por agressões aumentaram 26,4\% entre 2000 e 2010 (Tabela 5). Neste último ano, as internações mais frequentes no grupo das agressões foram decorrentes de objeto contundente $(28,3 \%)$, de disparo de arma de fogo de mão $(26,0 \%)$ e de objeto cortante ou penetrante $(19,8 \%)$, que somadas representaram mais de $74,0 \%$ das hospitalizações por agressões no ano 2010 . Importante destacar também que a faixa etária mais jovem (20 a 29 anos) foi a que apresentou a maior proporção das internações pelo referido grupo de causas na Bahia em 2010 (Gráfico 2).

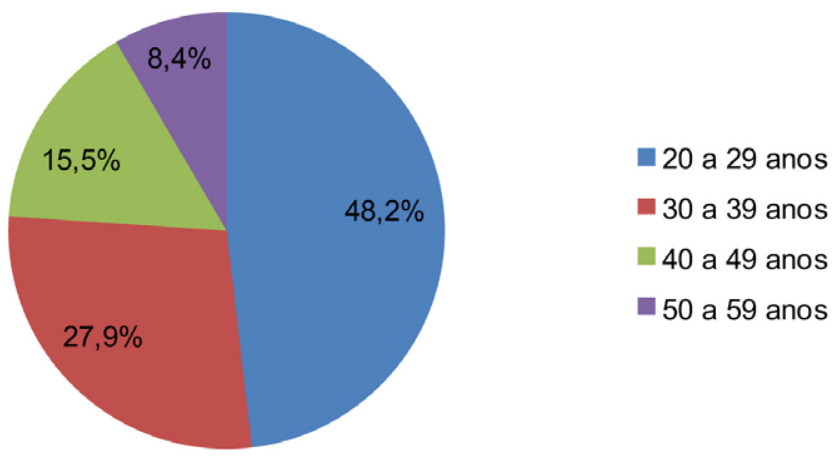

Gráfico 2. Proporção de internações hospitalares* por agressões, segundo faixa etária. Bahia, 2010.

*Internação hospitalares para homens na faixa etária de 20 a 59 anos. Fonte: Sesab/Suvisa/DIS-SIH-SUS.

\section{Internações por doenças do aparelho digestivo}

O grupo de doenças do aparelho digestivo foi o segundo grupo de causas de internaçães da população masculina de 20 a 59 anos na Bahia no ano de 2010. Entretanto é importante observar a redução da taxa de internação das doenças digestivas em 22,6\% quando se compara os anos de 2000 e 2010 (Tabela 1). $\mathrm{Na}$ análise por decênios da faixa etária, no mesmo período, deve-se registrar que houve uma redução mais significativa das taxas de internação no grupo de homens de 30 a 39 anos $(-28,2 \%)$, seguida pela diminuição entre os de 20 a 29 anos (- $27,9 \%$ ) - Gráfico 3. 


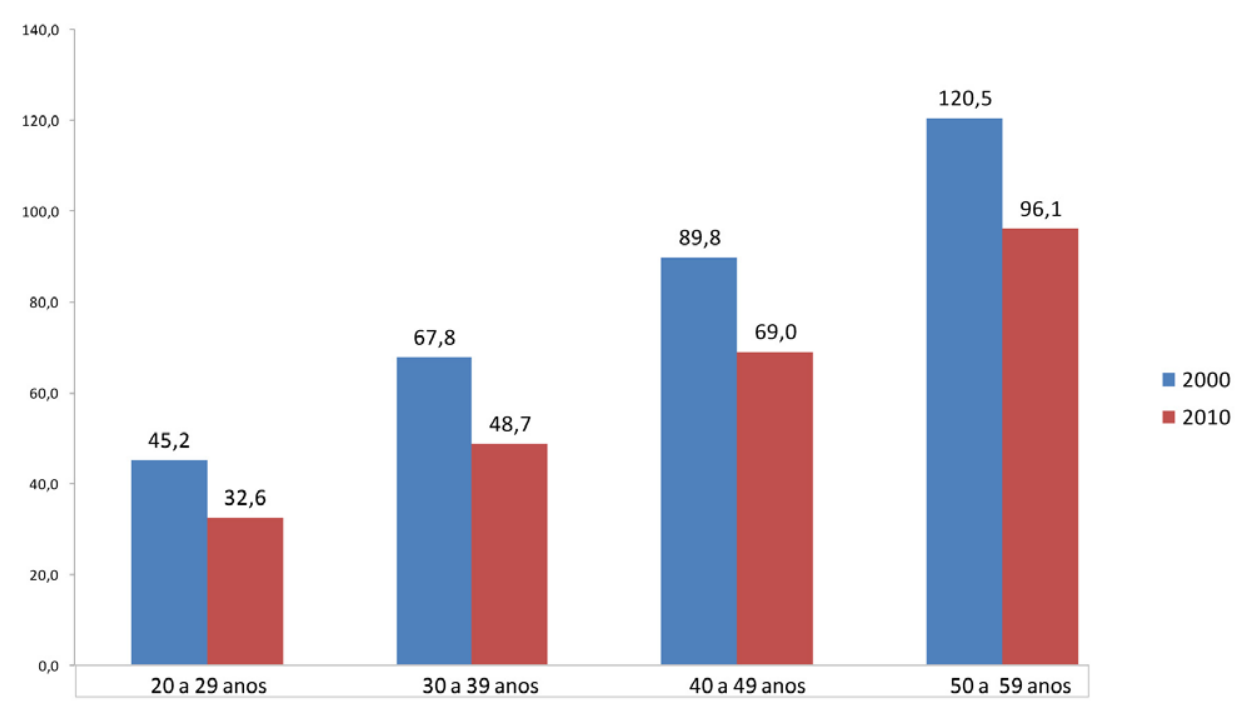

Gráfico 3. Taxas* de internação hospitalar por doenças do aparelho digestivo em homens, segundo faixa etária. Bahia, 2000 e 2010.

*Taxas de internação calculadas para 10.000 habitantes, para a faixa etária de 20 a 59 anos.

Analisando as taxas de internação das doenças do aparelho digestivo segundo NRS, observou-se uma tendência de redução na maioria dos NRS, com exceção do Sudoeste e do Oeste, que apresentaram, respectivamente, aumentos percentuais de 8,6 e 6,2

Tabela 6. Taxas* de internação e variação percentual** por doenças do aparelho digestivo em homens, segundo NRS. Bahia, 2000 e 2010.

\begin{tabular}{lccc}
\hline \multicolumn{1}{c}{ NRS } & \multicolumn{2}{c}{ Ano } & Variação percentual (\%) \\
\hline Centro-Norte & 2000 & 2010 & $-7,9$ \\
Sul & 88,0 & 81,0 & $-34,6$ \\
Norte & 104,3 & 68,2 & $-14,7$ \\
Sudoeste & 78,1 & 66,7 & 8,6 \\
Extremo Sul & 57,3 & 62,2 & $-15,5$ \\
Nordeste & 69,8 & 59,0 & $-5,7$ \\
Centro-Leste & 58,5 & 55,1 & $-28,7$ \\
Oeste & 71,3 & 50,8 & 6,2 \\
Leste & 47,5 & 50,5 & $-33,8$ \\
\hline
\end{tabular}

Fonte: Sesab/Suvisa/DIS-SIH-SUS.

*Taxas de internação calculadas para 10.000 habitantes, para a faixa etária de 20 a 59 anos.

**Variação percentual entre os anos 2000 e 2010.

No período de 2000 a 2010 ocorreu a redução das taxas de: hérnia inguinal, gastrite e duodenite, fibrose e cirrose hepáticas, colecistite e de outras doenças do aparelho digestivo. (Tabela 7).

Tabela 7. Taxas* de internação e variação percentual** por doenças do aparelho digestivo em homens. Bahia, 2000 e 2010.

\begin{tabular}{lccc}
\hline \multicolumn{1}{c}{ Doenças do aparelho digestivo } & Ano & Variação percentual (\%) \\
\hline Hernia inguinal & 2000 & 2010 & $-27,2$ \\
Gastrite e duodenite & 20,3 & 14,8 & $-29,5$ \\
Outras doencas do aparelho digestivo & 8,4 & 5,9 & $-36,0$ \\
Fibrose e cirrose hepaticas & 6,5 & 4,2 & $-68,4$ \\
Colecistite & 3,5 & 1,1 & $-35,3$ \\
Hernia umbilical & 2,9 & 1,9 & 37,9 \\
\hline
\end{tabular}

Fonte: Sesab/Suvisa/DIS-SIH-SUS.

*Taxas de internação calculadas para 10.000 habitantes, para a faixa etária de 20 a 59 anos.

**Variação percentual entre os anos 2000 e 2010. 
Mesmo considerando os importantes avanços na redução das mortes pelas DIP, estas são a terceira causa de internações dos homens adultos no Estado da Bahia em 2010 (Tabela 1). No Gráfico 4 é possível observar o aumento das internações por este grupo de causas em todas as faixas etárias pesquisadas.

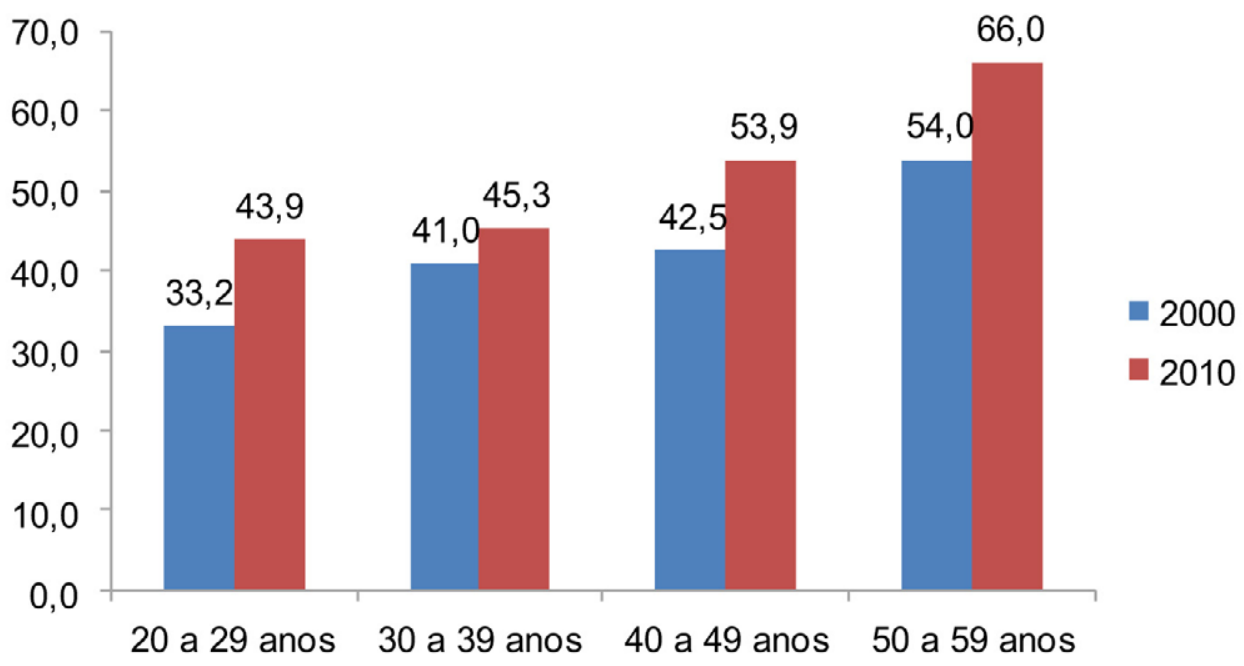

Gráfico 4. Taxas* de internação hospitalar por doenças infecciosas e parasitárias em homens. Bahia, 2000 e 2010.

Fonte: Sesab/Suvisa/DIS-SIH-SUS.

*Taxas de internação calculadas para 10.000 habitantes, para a faixa etária de 20 a 59 anos.

Na Tabela 8, pode ser observado o aumento da taxa de internações hospitalares por DIP em quase todos os NRS, com exceção do Norte que apresentou uma redução $(-13,8 \%)$.

Tabela 8. Taxas* de internação e variação percentual** por doenças infecciosas e parasitárias em homens, segundo NRS. Bahia, 2000 e 2010.

\begin{tabular}{lccc}
\hline \multicolumn{1}{c}{ NRS } & Ano & & \\
& 2000 & 2010 & Variação percentual (\%) \\
\hline Centro-Norte & 73,0 & 100,8 & 38,1 \\
Sul & 66,8 & 79,8 & 19,6 \\
Centro-Leste & 37,8 & 59,0 & 55,9 \\
Sudoeste & 42,6 & 54,6 & 28,3 \\
Nordeste & 45,1 & 49,2 & 9,1 \\
Oeste & 29,8 & 49,1 & 64,6 \\
Norte & 49,9 & 43,0 & $-13,8$ \\
Extremo Sul & 35,1 & 42,1 & 19,9 \\
Leste & 23,3 & 28,7 & 23,2 \\
\hline
\end{tabular}

Fonte: Sesab/Suvisa/DIS-SIH-SUS.

*Taxas de internação calculadas para 10.000 habitantes, para a faixa etária de 20 a 59 anos.

**Variação percentual entre os anos 2000 e 2010.

Apesar da redução da taxa de internação por doenças infecciosas intestinais (-10,9\%) entre 2000 e 2010, esta continuou como a mais frequente entre as internações por DIP. As maiores reduções no período foram observadas nas taxas de septicemia estreptocócica $(-92,3 \%)$ e doenças por HIV resultando em outras doenças $(-92,3 \%)$. Em contraste, a taxa de internação por dengue ( $3^{a}$ mais frequente entre as DIP em 2010$)$ apresentou um crescimento de mais de 6 vezes no período analisado (Tabela 9). 
Tabela 9. Taxas* de internação e variação percentual** por doenças infecciosas e parasitárias em homens. Bahia, 2000 e 2010.

\begin{tabular}{lccc}
\hline Doenças infecto parasitárias & 2000 & 2010 & $\begin{array}{c}\text { Variação } \\
\text { percentual (\%) }\end{array}$ \\
\hline Doenças infecciosas intestinais & 22,9 & 20,4 & $-10,9$ \\
Infecciosas bacterianas de local NE & 3,9 & 8,4 & 117,1 \\
Dengue & 0,9 & 6,4 & 616,4 \\
Erisipela & 0,7 & 2,1 & 200,0 \\
Tuberculose & 1,4 & 2,0 & 42,9 \\
Septicemia estreptocócica & 1,3 & 0,1 & $-92,3$ \\
Doença para HIV resultando em outras & 1,3 & 0,1 & $-92,3$ \\
doenças & 0,4 & 0,2 & $-50,0$ \\
Hanseníase & & &
\end{tabular}

Fonte: Sesab/Suvisa/DIS-SIH-SUS.

*Taxas de internação calculadas para 10.000 habitantes, para a faixa etária de 20 a 59 anos.

\section{Internações por doenças do aparelho circulatório}

O grupo de doenças do aparelho circulatório foi o quarto grupo de causas de internações da população masculina de 20 a 59 anos na Bahia no ano de 2010. Apesar da redução das taxas de internação em todas as faixas etárias no período de 2000 a 2010 , observa-se que o comportamento das taxas de internações por DAC é de aumento com o envelhecimento da população (Gráfico 5).

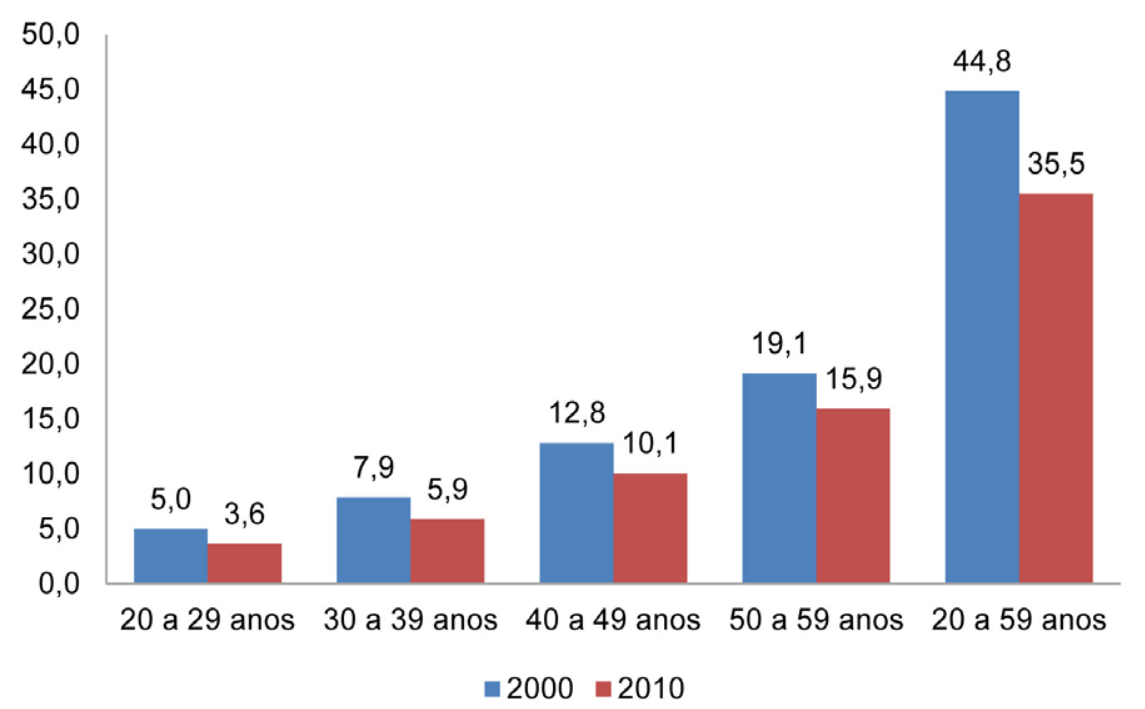

Gráfico 5. Taxas* de internação hospitalar por doenças do aparelho circulatório em homens, segundo faixa etária. Bahia, 2000 e 2010.

Fonte: Ministério da Saúde/Datasus-SIH-SUS; Sesab/Suvisa/DIS.

*Taxas de internação calculadas para 10.000 habitantes, para a faixa etária de 20 a 59 anos.

Na Tabela 10 é possível observar que como o Brasil, a Bahia apresentou decréscimo das taxas de internação por DAC, mas no referido estado a redução foi maior para todas as faixas etárias.

Tabela 10. Taxas* de internação hospitalar e variação percentual** por Doenças do Aparelho Circulatório, por faixa etária, em homens. Bahia e Brasil, 2000 e 2010.

\begin{tabular}{lcccccc}
\hline \multirow{2}{*}{ Faixa etária } & \multicolumn{2}{c}{ Bahia } & \multirow{2}{*}{ Variação \% } & 2000 & 2010 & \multirow{2}{*}{ Variação \% } \\
\hline 20 a 29 anos & 13,1 & 10,5 & $-19,9$ & 11,3 & 10,0 & $-10,9$ \\
30 a 39 anos & 28,1 & 20,8 & $-25,9$ & 25,7 & 21,2 & $-17,7$ \\
40 a 49 anos & 63,0 & 45,9 & $-27,0$ & 70,0 & 55,4 & $-20,8$ \\
50 a 59 anos & 141,2 & 104,2 & $-26,2$ & 170,4 & 138,2 & $-18,9$ \\
20 a 59 anos & 44,8 & 35,5 & $-20,8$ & 50,8 & 44,9 & $-11,6$ \\
\hline
\end{tabular}

Fonte: Ministério da Saúde/Datasus-SIH-SUS; Sesab/Suvisa/DIS.

*Taxas de internação calculadas para 10.000 habitantes, para a faixa etária de 20 a 59 anos.

**Variação percentual entre os anos 2000 e 2010. 
Percebe-se que apesar do NRS Centro-Norte apresentar as taxas de internações mais altas por DAC em 2000 e 2010, foi nele onde observou-se a maior redução das taxas de internações $(-27,7)$. No NRS Nordeste observaram-se as taxas mais baixas e também a menor redução no período (de 23,6 para 23,4, variação de $-1,2 \%)$ - Tabela 11 .

Tabela 11. Taxas* de internação hospitalar e variação percentual** das Doenças do Aparelho Circulatório em homens, segundo NRS. Bahia, 2000 e 2010.

\begin{tabular}{lccc}
\hline NRS & 2000 & 2010 & Variação \% \\
\hline Centro-Norte & 79,0 & 57,1 & $-27,7$ \\
Sul & 64,3 & 49,8 & $-22,5$ \\
Sudoeste & 44,1 & 40,5 & $-8,1$ \\
Extremo Sul & 41,8 & 34,6 & $-17,2$ \\
Leste & 41,0 & 32,3 & $-21,3$ \\
Oeste & 36,0 & 31,8 & $-11,5$ \\
Centro-Leste & 41,4 & 31,5 & $-23,9$ \\
Norte & 33,9 & 29,2 & $-13,8$ \\
Nordeste & 23,6 & 23,4 & $-1,2$ \\
\hline
\end{tabular}

Fonte: Sesab/Suvisa/DIS-SIH-SUS.

*Taxas de internação calculadas para 10.000 habitantes, para a faixa etária de 20 a 59 anos.

**Variação percentual entre os anos 2000 e 2010.

Dentre as internações por DAC destaca-se a insuficiência cardíaca, que apresentou as maiores taxas em 2000 e 2010 , e também a maior redução percentual $(-57,9 \%)$ no referido período. Em contraste, as taxas de internação por doenças isquêmicas do coração aumentaram 66,7\% (Tabela 12).

Tabela 12. Taxas* de internações hospitalar e variação percentual** por doenças do aparelho circulatório em homens. Bahia, 2000 e 2010.

\begin{tabular}{lccc}
\hline Doenças do Aparelho Circulatório & 2000 & 2010 & Variação \% \\
\hline Doenças hipertensivas(110 - I15) & 6,9 & 5,6 & $-18,8$ \\
Doenças isquêmicas do coração (120 - 125) & 3,0 & 5,0 & 66,7 \\
Insuficiência cardíaca (I50) & 19,5 & 8,2 & $-57,9$ \\
Doenças cerebrovasculares (160 - 169) & 5,1 & 4,8 & $-5,9$ \\
\hline
\end{tabular}

Fonte: Sesab/Suvisa/DIS-SIH-SUS.

*Taxas de internação calculadas para 10.000 habitantes, para a faixa etária de 20 a 59 anos.

**Variação percentual entre os anos 2000 e 2010.

\section{Internações por doenças do aparelho respiratório (DAR)}

No ano 2000, na faixa etária de 20 a 59 anos, o grupo das DAR foi o mais prevalente entre as causas de internações na Bahia, porém houve uma significativa redução $(-50,8 \%)$ e o grupo de DAR passou a ser quinto grupo de causas de internações na Bahia no ano de 2010 (Tabela 1). Além disso, observou-se que em todas as faixas etárias houve redução no comparativo de 2000 e 2010, tanto na Bahia como no Brasil (Tabela 13).

Tabela 13. Taxas* de internação hospitalar e variação percentual** por Doenças do Aparelho Respiratório, por faixa etária, em homens. Bahia e Brasil, 2000 e 2010.

\begin{tabular}{|c|c|c|c|c|c|c|}
\hline \multirow{2}{*}{ Faixa etária } & \multicolumn{2}{|c|}{ Bahia } & \multirow{2}{*}{ Variação \% } & \multicolumn{2}{|c|}{ Brasil } & \multirow{2}{*}{ Variação \% } \\
\hline & 2000 & 2010 & & 2000 & 2010 & \\
\hline 20 a 29 anos & 55,9 & 28,2 & $-49,6$ & 38,1 & 22,9 & $-40,0$ \\
\hline 30 a 39 anos & 59,9 & 28,8 & $-51,9$ & 42,6 & 25,5 & $-40,0$ \\
\hline 40 a 49 anos & 78,3 & 36,5 & $-53,4$ & 59,6 & 35,8 & $-39,9$ \\
\hline 50 a 59 anos & 130,5 & 60,8 & $-53,5$ & 112,9 & 63,1 & $-44,1$ \\
\hline 20 a 59 anos & 71,7 & 35,2 & $-51,0$ & 54,7 & 33,3 & $-39,1$ \\
\hline
\end{tabular}

Fonte: Ministério da Saúde/Datasus-SIH-SUS

*Taxas de internação calculadas para 10.000 habitantes, para a faixa etária de 20 a 59 anos.

**Variação percentual entre os anos 2000 e 2010. 
Na maioria dos NRS também ocorreram significativas reduções, entretanto essas taxas continuam, ainda, elevadas (Tabela 14).

Tabela 14. Taxas* de internações hospitalar e variação percentual** das Doenças do Aparelho Respiratório em homens, segundo NRS, Bahia, 2000 e 2010.

\begin{tabular}{lccc}
\hline NRS & 2000 & 2010 & Variação\% \\
\hline Centro-Norte & 181,6 & 80,7 & $-55,5$ \\
Sul & 125,1 & 68,0 & $-45,7$ \\
Oeste & 113,1 & 51,9 & $-54,1$ \\
Sudoeste & 83,7 & 40,7 & $-51,3$ \\
Centro-Leste & 66,1 & 34,6 & $-47,7$ \\
Extremo Sul & 77,6 & 32,3 & $-58,4$ \\
Nordeste & 42,6 & 31,0 & $-27,4$ \\
Norte & 67,8 & 29,7 & $-56,2$ \\
Leste & 25,0 & 13,8 & $-44,9$ \\
\hline
\end{tabular}

Fonte: Sesab/Suvisa/DIS-SIH-SUS.

*Taxas de internação calculadas para 10.000 habitantes, para a faixa etária de 20 a 59 anos.

**Variação percentual entre os anos 2000 e 2010.

As mais frequentes causas de internação por doenças respiratórias foram: as pneumonias, a asma, a insuficiência respiratória, algumas doenças obstrutivas e a influenza. Todas as causas mencionadas apresentaram uma diminuição das taxas, exceto a influenza (Tabela 15).

Tabela 15. Taxas* de internações hospitalar e variação percentual** por doenças do aparelho respiratório em homens. Bahia, 2000 e 2010.

\begin{tabular}{lccc}
\hline Doenças & 2000 & 2010 & Variação \\
\hline Pneumonia & 32,2 & 16,9 & $-47,6$ \\
Asma & 25,0 & 7,7 & $-69,2$ \\
Insuficiência respiratória & 8,7 & 2,3 & $-74,0$ \\
Outras doenças pulmonares obstrutivas crônicas & 5,8 & 1,7 & $-70,9$ \\
Influenza & 0,0 & 1,1 & \\
\hline
\end{tabular}

Fonte: Sesab/Suvisa/DIS-SIH-SUS.

*Taxas de internação calculadas para 10.000 habitantes, para a faixa etária de 20 a 59 anos.

**Variação percentual entre os anos 2000 e 2010.

\section{Internações por doenças do aparelho geniturinário}

As doenças e distúrbios do aparelho geniturinário foram a sexta causa de internações da população masculina adulta na Bahia no ano de 2010. Observou-se a redução nas taxas de internação por estas doenças em todas as faixas etárias pesquisadas no período de 2000 a 2010 na Bahia, seguindo a mesma tendência nacional (Tabela 16).

Tabela 16. Taxas* de internação hospitalar e variação percentual** por Doenças do Aparelho Geniturinário, por faixa etária, em homens. Bahia e Brasil, 2000 e 2010.

\begin{tabular}{|c|c|c|c|c|c|c|}
\hline \multirow{2}{*}{ Faixa etária } & \multicolumn{2}{|c|}{ Bahia } & \multirow{2}{*}{ Variaçãa \% } & \multicolumn{2}{|c|}{ Brasil } & \multirow{2}{*}{ Variação \% } \\
\hline & 2000 & 2010 & & 2000 & 2010 & \\
\hline 20 a 29 anos & 20,5 & 15,2 & $-35,2$ & 18,9 & 14,7 & $-22,2$ \\
\hline 30 a 39 anos & 28,0 & 20,5 & $-37,0$ & 23,7 & 19,8 & $-16,5$ \\
\hline 40 a 49 anos & 29,0 & 26,7 & $-8,8$ & 28,1 & 25,6 & $-8,8$ \\
\hline 50 a 59 anos & 53,2 & 37,1 & $-43,4$ & 45,1 & 40,1 & $-11,1$ \\
\hline 20 a 59 anos & 28,8 & 22,5 & $-27,7$ & 26,0 & 22,8 & $-12,1$ \\
\hline
\end{tabular}

Fonte: Ministério da Saúde/Datasus-SIH-SUS; Sesab/Suvisa/DIS.

*Taxas de internação calculadas para 10.000 habitantes, para a faixa etária de 20 a 59 anos.

**Variação percentual entre os anos 2000 e 2010. 
O NRS Centro-Norte liderou as taxas de internações por doenças geniturinárias em 2000 e em 2010 . Foi observada uma tendência de diminuição na maioria dos NRS, com destaque para o Sul e o Extremo Sul, que apresentaram reduções de $45,4 \%$ e $40,4 \%$, respectivamente (Tabela 17 ).

Tabela 17. Taxas* de internação hospitalar e variação percentual** por Doenças do Aparelho Geniturinário, por NRS, em homens. Bahia, 2000 e 2010.

\begin{tabular}{lccc}
\hline NRS & 2000 & 2010 & Variação \% \\
\hline Centro-Norte & 50,7 & 45,6 & $-10,1$ \\
Norte & 24,0 & 26,6 & 11,0 \\
Sudoeste & 29,4 & 25,6 & $-12,8$ \\
Centro-Leste & 25,6 & 24,1 & $-5,6$ \\
Sul & 43,8 & 23,9 & $-45,4$ \\
Oeste & 18,6 & 21,5 & 15,3 \\
Nordeste & 27,3 & 19,0 & $-30,3$ \\
Extremo Sul & 29,6 & 17,6 & $-40,4$ \\
Leste & 22,4 & 16,8 & $-24,9$ \\
\hline
\end{tabular}

Fonte: Sesab/Suvisa/DIS-SIH-SUS.

*Taxas de internação calculadas para 10.000 habitantes, para a faixa etária de 20 a 59 anos.

**Variação percentual entre os anos 2000 e 2010.

Dentre as taxas de internação por doenças do aparelho geniturinário, destaca-se o aumento significativo por outros transtornos do trato urinário (de 1,7 para 4,1, variação de 146,4\%) - Tabela 18.

Tabela 18. Taxas* de internação hospitalar e variação percentual** por Doenças do Aparelho Geniturinário, em homens. Bahia, 2000 e 2010.

\begin{tabular}{lccc} 
Doenças do Aparelho Geniturinário & 2000 & 2010 & Variação \% \\
\hline Outros transtornos do trato urinário & 1,7 & 4,1 & 146,4 \\
Calculose do rim e do ureter & 2,9 & 2,6 & $-8,8$ \\
Hidrocele e espermatocele & 2,6 & 2 & $-20,1$ \\
Insuficiência renal crônica & 1,6 & 1,7 & 6,7 \\
Nefrite tubulo-intersticial crônica & 1,8 & 1,6 & $-9,2$ \\
\hline
\end{tabular}

Fonte: Sesab/Suvisa/DIS-SIH-SUS.

*Taxas de internação calculadas para 10.000 habitantes, para a faixa etária de 20 a 59 anos.

**Variação percentual entre os anos 2000 e 2010.

\section{Internações por neoplasias}

Neste capítulo estão incluídas as neoplasias malignas e benignas de diferentes órgãos. No Estado da Bahia, em todas as faixas etárias pesquisadas, houve aumento da taxa de internação por neoplasia, com exceção para a de 20 a 29 anos, que apresentou redução. Observou-se ainda, que a faixa etária mais acometida por neoplasias foi a de 50 a 59 anos (Tabela 19).

Tabela 19. Taxas* de internação hospitalar e variação percentual** por Neoplasia, por faixa etária, em homens. Bahia e Brasil, 2000 e 2010.

\begin{tabular}{|c|c|c|c|c|c|c|}
\hline \multirow{2}{*}{ Faixa etária } & \multicolumn{2}{|c|}{ Bahia } & \multirow{2}{*}{ Variação \% } & \multicolumn{2}{|c|}{ Brasil } & \multirow{2}{*}{ Variação \% } \\
\hline & 2000 & 2010 & & 2000 & 2010 & \\
\hline 20 a 29 anos & 6,0 & 5,2 & $-15,4$ & 6,1 & 7,3 & 19,7 \\
\hline 30 a 39 anos & 8,1 & 8,5 & 4,7 & 8,2 & 9,6 & 17,1 \\
\hline 40 a 49 anos & 13,3 & 16,4 & 18,9 & 17,6 & 22,1 & 25,6 \\
\hline 50 a 59 anos & 24,9 & 39,5 & 37,0 & 38,9 & 55,4 & 42,4 \\
\hline 20 a 59 anos & 10,6 & 13,8 & 23,2 & 13,9 & 19,4 & 39,6 \\
\hline
\end{tabular}

Fonte: Ministério da Saúde/Datasus-SIH-SUS; Sesab/Suvisa/DIS.

*Taxas de internação calculadas para 10.000 habitantes, para a faixa etária de 20 a 59 anos.

**Variação percentual entre os anos 2000 e 2010. 
Com relação aos NRS, ressalva-se que no comparativo dos anos de 2000 e 2010 embora se observe aumento da taxa na maioria dos NRS, com destaque para o Centro-Norte e o Oeste, que apresentaram, respectivamente, aumento percentual de 136,4 e 118,3. Apenas o NRS Sul apresentou redução (taxa de 19,9 para 14,4). Destaca-se ainda, a situação do NRS Leste, que embora tenha apresentado um crescimento da taxa menor que a maioria dos NRS da Bahia, apresentou as maiores taxas de internação por neoplasia tanto em 2000, quanto em 2010 (Tabela 20).

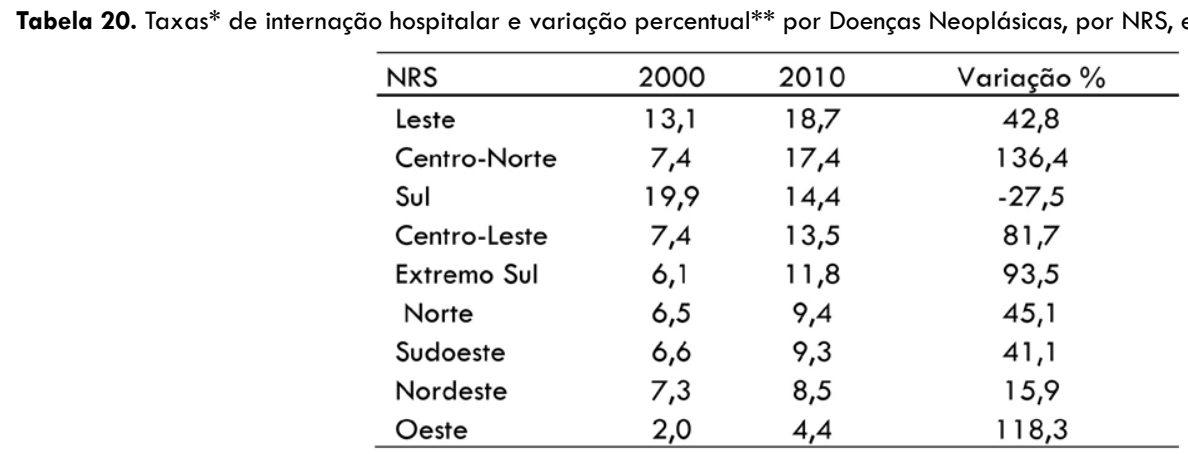

Fonte: Sesab/Suvisa/DIS-SIH-SUS.

*Taxas de internação calculadas para 10.000 habitantes, para a faixa etária de 20 a 59 anos.

**Variação percentual entre os anos 2000 e 2010.

Nos anos de 2000 e 2010, observou-se o aumento na taxa de algumas doenças neoplásicas, tais como: hemangioma e linfangioma, neoplasia maligna da próstata, neoplasia maligna do estomago, neoplasia maligna do esôfago, e neoplasia maligna da laringe (Tabela 21 ).

Tabela 21. Taxas* de internação hospitalar e variação percentual** por Doenças Neoplásicas, em homens. Bahia, 2000 e 2010.

\begin{tabular}{lccc} 
Doenças neoplásticas & 2000 & 2010 & Variação \% \\
\hline Hemangioma e linfangioma & 0,7 & 1,1 & 57 \\
Neoplasia maligna do estômago & 0,1 & 0,7 & 600 \\
Neoplasia maligna do esôfago & 0,3 & 0,6 & 100 \\
Neoplasia maligna da próstata & 0,3 & 0,5 & 66,7 \\
Outras neoplasias benignas da pele & 0,2 & 0,5 & 150 \\
Outras neoplasias malignas da pele & 0,6 & 0,5 & $-23,2$ \\
Neoplasia maligna da laringe & 0,2 & 0,4 & 100 \\
Neoplasia maligna do pênis & 0,1 & 0,2 & 100 \\
\hline Fonte: Sesab/Suvisa/DIS-SIH-SUS. & & & \\
*Taxas de internação calculadas para 10.000 habitantes, para a faixa etária de 20 a 59 anos. \\
**Variação percentual entre os anos 2000 e 2010.
\end{tabular}

\section{Internações por transtornos mentais e comportamentais}

Os transtornos mentais e comportamentais, oitava causa de internação da população masculina adulta no Estado da Bahia no ano de 2010, são definidos como condições caracterizadas por alterações mórbidas do modo de pensar e/ou do humor (emoções), e/ou por alterações mórbidas do comportamento associadas à angústia expressiva e/ou deterioração do funcionamento psíquico global.

No Estado da Bahia, observou-se a redução da taxa de internação por transtornos mentais, seguindo a mesma tendência de diminuição observada para o Brasil (Tabela 22). 
Tabela 22. Taxas* de internação hospitalar e variação percentual** por Transtornos Mentais e Comportamentais, por faixa etária, em homens. Bahia e Brasil, 2000 e 2010.

\begin{tabular}{|c|c|c|c|c|c|c|}
\hline \multirow{2}{*}{ Faixa etária } & \multicolumn{2}{|c|}{ Bahia } & \multirow{2}{*}{ Variação \% } & \multicolumn{2}{|c|}{ Brasil } & \multirow{2}{*}{ Variação \% } \\
\hline & 2000 & 2010 & & 2000 & 2010 & \\
\hline 20 a 29 anos & 22,4 & 10,5 & $-53,1$ & 35,8 & 26,0 & $-27,4$ \\
\hline 30 a 39 anos & 39,5 & 15,3 & $-61,3$ & 65,2 & 33,7 & $-48,3$ \\
\hline 40 a 49 anos & 44,6 & 14,7 & $-67,0$ & 73,5 & 36,9 & $-49,8$ \\
\hline 50 a 59 anos & 28,9 & 11,8 & $-59,2$ & 59,6 & 28,7 & $-51,8$ \\
\hline 20 a 59 anos & 32,6 & 13,0 & $-60,1$ & 55,9 & 31,1 & $-44,4$ \\
\hline
\end{tabular}

Fonte: Sesab/Suvisa/DIS-SIH-SUS.

*Taxas de internação calculadas para 10.000 habitantes, para a faixa etária de 20 a 59 anos.

**Variação percentual entre os anos 2000 e 2010.

Ao analisar as taxas por NRS, verificou-se que o Norte apresentou um aumento $(54,0 \%)$ em contraste com a tendência observada em todo o restante dos NRS do Estado da Bahia (Tabela 23).

Tabela 23. Taxas* de internação hospitalar e variação percentual** por Transtornos Mentais e Comportamentais, por NRS, em homens. Bahia, 2000 e 2010.

\begin{tabular}{lccc} 
NRS & 2000 & 2010 & Variação \% \\
\hline Sul & 56,8 & 38,3 & $-32,6$ \\
Centro-Leste & 66,1 & 34,6 & $-47,7$ \\
Norte & 19,8 & 30,5 & 54 \\
Leste & 128,9 & 27 & $-79,1$ \\
Sudoeste & 20,6 & 8,6 & $-85,3$ \\
Extremo Sul & 14,3 & 6,4 & $-55,2$ \\
Nordeste & 36,5 & 4,5 & $-87,7$ \\
Centro-Oeste & 29,4 & 3,7 & $-87,4$ \\
Oeste & 6,6 & 2,1 & $-68,2$ \\
\hline
\end{tabular}

Fonte: Sesab/Suvisa/DIS-SIH-SUS.

*Taxas de internação calculadas para 10.000 habitantes, para a faixa etária de 20 a 59 anos.

**Variação percentual entre os anos 2000 e 2010.

Dentre as internações por transtornos mentais e comportamentais destacam-se as expressivas reduções das taxas para: psicose não-orgânica, esquizofrenia e os transtornos mentais e comportamentais devido ao uso de álcool (Tabela 24). Tal cenário ocorreu de forma semelhante ao analisar por faixa etária (20 a 29,30 a 39 , 40 a 49 e 50 a 59 ).

Tabela 24. Taxas* de internação hospitalar e variação percentual** por Transtornos Mentais e Comportamentais, em homens. Bahia, 2000 e 2010.

\begin{tabular}{lccc} 
Transtornos mentais e comportamentais & 2000 & 2010 & Variação \% \\
\hline Esquizofrenia & 28,8 & 8,7 & $-69,9$ \\
Transt. Mentais e comportamentais devido ao uso álcool & 10 & 3,2 & $-67,8$ \\
Psicose não-orgânica NE & 15,5 & 3,1 & $-80,1$ \\
Retardo mental moderado & 1 & 0,7 & $-28,5$ \\
Retardo mental grave & 1,4 & 0,6 & -57 \\
\hline
\end{tabular}

Fonte: Sesab/Suvisa/DIS-SIH-SUS.

*Taxas de internação calculadas para 10.000 habitantes, para a faixa etária de 20 a 59 anos.

**Variação percentual entre os anos 2000 e 2010.

\section{DISCUSSÃO}

Uma das formas de se pesquisar o perfil de morbidade de uma população é estudar as causas de internação hospitalar. O SIH/SUS é um dos sistemas de informação em saúde mais utilizado em análises da situação de saúde por morbidade hospitalar. Entretanto, devemos observar algumas limitações: a cobertura irregular entre as grandes regiões e nos estados do país, a confiabilidade das informações da $\mathrm{AlH}$, a suspeita de fraudes 
nas possíveis tentativas de aumento do pagamento financeiro, além da cobertura restrita à rede assistencial e conveniada ao SUS e não identifica as possíveis reinternações ${ }^{11}$.

É fundamental para as políticas de saúde desenvolvidas no Brasil o desenvolvimento de estudos e pesquisas relacionadas ${ }^{3,4,5,6}$ à atenção da saúde masculina, assim como os fatores de riscos para o adoecimento e comportamentos danosos à saúde gerados pelas diversas concepções sociais, culturais, econômicas e políticas. Neste contexto um aspecto evidente é, ainda, a valorização de um discurso hegemônico que configura ideais de masculinidade, favorecendo o descuido com a saúde em cenários de ampla complexidade inclusive no que se refere ao acesso, acolhimento e vínculo nos serviços de saúde, os quais podem ser melhor entendidos com outros estudos ${ }^{10,12}$ que abordam dimensões dos problemas deste grupo a partir da última década e que podem ser melhor compreendidos também com informações de morbidade avançada.

Os grupos de causas de hospitalização mais frequentes na população masculina de 20 a 59 anos do estado da Bahia no ano de 2010 foram, respectivamente: causas externas, doenças do aparelho digestivo, infecto parasitárias, do aparelho circulatório e do respiratório. É evidente o aumento das internações com o aumento da idade em todas as faixas etárias, de todas as principais causas com exceção das causas externas/lesões, tendência já observada no ano 2000.

Como Laurenti ${ }^{13}$ já demonstrou existem quatro grupos de doenças que oscilam entre os países das Américas, mas sempre estão presentes como principais causas de internação e morte de indivíduos do sexo masculino. Constituem um dos aspectos da vulnerabilidade dos homens diante de agravos: 1) neoplasias malignas (cânceres de estômago, pulmão e próstata); 2) as doenças isquêmicas do coração; 3 ) as doenças cerebrovasculares e 4) as causas externas (destacando-se os acidentes de carro e os homicídios). No dizer do autor certos agravos são mais bem compreendidos como produto do comportamento masculino no ambiente social; outros, na relação trabalho-adoecimento, como produto do modo como se dá o exercício das masculinidades e outros, ainda, como é o caso dos homicídios, em contraste e compondo com a violência doméstica, têm uma face de sua compreensão dada como comportamento violento de gênero ${ }^{11,13}$.

Observa-se uma tendência de crescimento na Bahia e no Brasil, entre 2000 e 2010 , para as taxas de internação por causas externas nos homens $(20$ a 59 anos), inclusive em todas as faixas etárias desagregadas. Destaca-se ainda que as maiores taxas por 10.000 habitantes foram identificadas para os adultos jovens, com idade de 20 a 29 na Bahia $(79,5)$ e no Brasil $(91,5)$. Outros estudos apontam tendência semelhante ao observado acima, porém com grupos mais jovens. Em 2014 na Bahia $^{14}$ para $\circ$ grupo de adolescentes $(10$ a 19 anos) do sexo masculino internados para tratamento de lesões decorrentes de causas externas, os quais tiveram risco aumentado em razão do aumento da idade, sendo a taxa de 44,9 para o grupo de 10 a 14 anos e de 71,7 dos 15 aos 19 anos. Mendes e Sala ${ }^{15}$ observaram que no estado de São Paulo as internações masculinas por lesões são bem superiores que as femininas em todas as faixas etárias a partir da infância, afetando principalmente os homens jovens e somente se igualando a partir dos 70 anos.

No comparativo por faixas etárias no Estado da Bahia nos anos de 2000 e 2010, detectam-se diferenças importantes. Enquanto nos adultos jovens, verifica-se maior frequência no atendimento de lesões por causas externas na faixa de 20 a 39 anos, seguida da presença de transtornos mentais, nas idades de 40 anos e mais, com proporções crescentes segundo as idades, surgem as doenças do aparelho circulatório, alternando posições, as doenças dos aparelhos respiratório e digestivo. Nos adultos jovens, entre 20 a 29 anos, verifica-se as maiores taxas de internações por causas externas, com destaque aos acidentes de trânsito envolvendo motociclistas.

Outras pesquisas ${ }^{16,17,18,19}$ afirmam que a faixa de 20 a 30 anos é a mais acometida nos acidentes envolvendo motociclistas. Lin e $\mathrm{Kraus}^{20}$ em uma revisão sistemática também revelou que as idades mais jovens são consideradas de maior risco para envolvimento em acidentes com motocicletas bem como associação com maior gravidade ou morte. Alguns autores ${ }^{20,21}$ observam que a taxa de internação no Sistema Único de Saúde (SUS) por 
acidentes de trânsito é de 64 internações para cada 100 mil habitantes, com média de 98 mortes por dia.

O elevado índice de acidentes com motociclistas pode estar relacionado ao número elevado da frota de motocicletas no país, tanto em áreas urbanas e como rurais. A sua utilização como instrumento de trabalho no mercado formal ou informal é referida por diversos autores como possível causa para o aumento do número de vítimas de motociclistas ${ }^{22,23}$.

Apesar das elevadas taxas de morbidade hospitalar dos homens, no comparativo entre 2000 e 2010, observou-se neste estudo redução nas taxas de internações hospitalares na maioria dos grandes grupos de causa na Bahia e no Brasil, apresentando, entretanto, aumento nas taxas de internação, além das causas externas, por neoplasias e doenças infectoparasitárias. No estado da Bahia, no ano de 2010, o grupo de doenças do aparelho digestivo foi o segundo grupo de causas de internações da população masculina de 20 a 59 anos. Entretanto, é importante observar a redução da taxa de internação das doenças digestivas em 22,6\% quando se compara os anos de 2000 e 2010, semelhante ao ocorrido com as doenças do aparelho circulatório, as doenças do aparelho respiratório e as doenças e distúrbios do aparelho geniturinário, além dos transtornos mentais e comportamentais, mas em oposição no mesmo período ao aumento das internações por doenças infecto parasitárias em todas as faixas etárias pesquisadas e às neoplasias que somente não sofreram aumento para a faixa etária de 20 a 29 anos.

A taxa de internação das doenças digestivas no comparativo dos anos de 2000 e 2010 no estado da Bahia, na análise por décadas, deve-se registrar que na faixa de 40 a 49 anos as mesmas figuram entre as doenças de maior morbidade em 2010 (taxa de 69,04 em 2010), nas faixas de 30 a 39 e 50 a 59 anos em segundo lugar e na faixa de 20 a 29 anos em terceiro, todas muito elevadas. No período citado, na faixa mais ampla de 20 a 59 anos, destaca-se o aumento na taxa de algumas doenças, tais como as doenças do apêndice (variação de $40,8 \%$ ) e em menor expressão os transtornos da vesícula biliar vias biliares pâncreas (variação de $4,5 \%$. Tal situação denota, entre outras mudanças de padrões dietéticos e de consumo de álcool, nas últimas décadas. Segundo Mendes e Sala 15, este fato também pode explicar os coeficientes maiores de internação masculina por doenças do aparelho digestivo, que incluem as doenças hepáticas relacionadas ao álcool.

No Estado da Bahia, no comparativo de 2000 e 2010 , também se observa crescimento das internações hospitalares por doenças infectoparasitárias, entre os homens e mulheres, em todas as faixas etárias, diferente do que acontece no Brasil. De acordo com os resultados obtidos, é importante ressaltar no mesmo período aumento expressivo nas taxas das doenças infectoparasitárias, (variação de 24,4\%), devido, principalmente, à dengue, à tuberculose e às infecções intestinais bacterianas. Estas são um sério problema de Saúde Pública e demonstram relação direta com a pobreza, diretamente associada com a exclusão social, insuficiente saneamento básico e a marginalização de parte da população submetida a más condições de vida, como moradia precária, desnutrição e dificuldades de acesso aos serviços e bens públicos.

Outro agravo de destaque é o Acidente Vascular Cerebral (AVC) por ser uma síndrome neurológica com grande prevalência em adultos e idosos, sendo também umas das maiores causas de mortalidade no mundo, e uma das principais causas de internações. Sua incidência é maior após os 65 anos, dobrando a cada década após os 55 anos de idade24,25.

Araújo (2008) destaca que uma série de fatores como hipertensão arterial, diabetes mellitus, doenças cardíacas, tabagismo, obesidade, tem contribuído diretamente para o aumento do número de internações pelo AVC. O Ministério da Saúde ${ }^{26}$ afirma que o AVC é uma das principais causas de morte e de sequelas no mundo e no Brasil. A doença cerebrovascular atinge 16 milhões de pessoas ao redor do globo a cada ano. Dessas, seis milhões morrem. Por isso, a Organização Mundial de Saúde (OMS) recomenda a adoção de medidas urgentes para a prevenção e tratamento da doença.

Outro estudo ${ }^{27}$ corrobora a mesma tendência de aumento de doenças do aparelho circulatório (DAC) nas últimas décadas e revela ainda, que a morbidade hospitalar mais frequente por DAC no 
estado da Bahia, no período de 2008 a 2013, foi a insuficiência cardíaca, na população mais idosa, seguida da hipertensão essencial primária em indivíduos mais jovens, além de que a maior ocorrência das internações hospitalares afetam mais os homens, apesar de que as mulheres vem apresentando um aumento das internações por DAC nesse estado.

No que se refere às internações por doenças do aparelho circulatório para homens e mulheres foi descrito no Estado de São Paulo, que os coeficientes de internação masculinos são semelhantes aos femininos até os 50 anos e posteriormente, os valores masculinos apresentam valores superiores que tendem a se acentuar até 80 anos ou mais, quando os coeficientes dos dois sexos voltam a se aproximar. Estes resultados podem indicar que as doenças circulatórias, quando se manifestam nos homens, apresentam maior gravidade, uma vez que o sexo masculino busca atendimento em serviços de saúde com menor frequência do que as mulheres, perdendo oportunidades de tratamentos precoces ${ }^{15}$.

Entretanto, na análise de Laurenti, Mello-Jorge e Gotlieb28, observa-se algumas diferenças no sexo masculino quando comparado com os estudos acima, pois na lista das principais causas que levaram à internação no Brasil em 2002, aparecem em primeiro lugar as doenças do aparelho respiratório, seguindo-se, com proporções semelhantes, doenças do aparelho circulatório, infecciosas, aparelho digestivo e lesões (causas externas).

Também o problema das doenças respiratórios tem correspondência com a pesquisa de Toyoshima, lto e Gouveia ${ }^{29}$, quando apontam que em São Paulo entre 1995 e 2000 houve decréscimo no número absoluto e na taxa de internações por doenças respiratórias totais em todas as idades, apesar da tendência de aumento importante, observada, principalmente entre os idosos para doença pulmonar obstrutiva crônica (DPOC). Neste estudo a população masculina foi mais afetada do que a feminina em todos os anos, por quaisquer causas estudadas, com exceção da asma e as pneumonias representaram a causa mais importante de hospitalizações, com $47 \%$ das internações.

As doenças respiratórias incluem uma ampla gama de patologias, como infecções respiratórias agudas, pneumonia, doenças pulmonares obstrutivas, doença pleural e pneumoconiose, além de malignidades do trato respiratório. As doenças respiratórias representam uma importante causa de morbidade e mortalidade em todo o mundo, sendo que as cinco principais contribuíram com $17,4 \%$ de todas as mortes e $13,3 \%$ dos anos de vida ajustados por incapacidade no ano 2000. As infecções do trato respiratório inferior, DPOC, TB e câncer de pulmão estão entre as dez principais causas de morte em todo o mundo ${ }^{30}$.

Por fim, é importante observar que no Estado da Bahia há o destaque da ocorrência de transtornos mentais em homens na faixa de 30 a 49 anos. Em São Paulo ${ }^{15}$, observa-se que os coeficientes masculinos de internação por transtornos mentais são bem superiores aos femininos em faixas etárias jovens, devido às internações por álcool e drogas e por substâncias psicoativas, que predominam bastante entre os homens.

A grande redução no número de internações hospitalares por transtornos mentais e comportamentais pode ser explicada pelo processo de diminuição de leitos hospitalares psiquiátricos e de desinstitucionalização de pessoas com longo histórico de internação, que se tornou uma política pública no Brasil a partir dos anos 90 e foi impulsionada em 2002 através de normatizações do Ministério da Saúde ${ }^{31}$.

\section{CONSIDERAÇÕES FINAIS}

Este estudo, juntamente com o conjunto de outras informações e instrumentos de planejamento produzido por outros setores, poderá apontar alguns dos principais problemas de morbidade hospitalar da população masculina a serem enfrentados pelos gestores e usuários nos diversos níveis do Sistema Único de Saúde (SUS).

Mesmo com as limitações impostas pelas informações de Internação Hospitalar, tanto na cobertura quanto na qualidade dos dados, esta publicação expressa, de forma satisfatória, a magnitude e a transcendência dos problemas de 
saúde da população masculina no estado da Bahia, proporcionando subsídios para as políticas públicas do Estado. Espera-se ainda que este estudo possa apoiar e reorientar as ações desenvolvidas pelo SUS no Estado, incorporando o conceito de necessidades de saúde, com flexibilidade de introdução de novas práticas no modelo assistencial.

Observa-se na faixa etária de 20 a 59 anos, na população masculina, o predomínio acentuado de internações em 2010 por causas externas, no grupo de homens, na Bahia, na rede hospitalar do SUS, no comparativo com o ano de 2000, embora as variações das doenças infectoparasitárias e neoplasias, no mesmo período, sejam mais elevadas que as causas externas.

A menor frequência dos homens aos serviços de atenção primária faz com que esse grupo populacional fique privado da proteção adequada à preservação de sua saúde, propiciando o uso de procedimentos desnecessários que poderiam ser evitados, caso a procura pela atenção houvesse ocorrido em momento oportuno. Por outro lado, a possível resistência masculina a atenção primária aumenta não somente a sobrecarga financeira da sociedade, mas, sobretudo, o sofrimento físico e emocional deles e de suas famílias na luta pela conservação da saúde e da qualidade de vida. Muitos agravos poderiam ser evitados, caso os homens realizassem com regularidade as medidas de prevenção primária.

Há necessidade de serem criadas redes de atendimento aos homens, além da qualificação dos profissionais de saúde da atenção básica e uma maior sensibilização por parte dos gestores Estaduais e Municipais.

O Estado da Bahia, apresenta importantes avanços no setor saúde na última década, embora necessite ampliar o planejamento e execução nas políticas específicas para os homens, as quais comportem as necessidades da população masculina, que, na maioria dos casos, depende inteiramente de tais ações públicas para os cuidados com a saúde.

\section{CONFLITOS DE INTERESSES}

Nenhum conflito financeiro, legal ou político envolvendo terceiros (governo, empresas e fundações privadas, etc.) foi declarado para nenhum aspecto do trabalho submetido (incluindo, mas não limitandose a subvenções e financiamentos, conselho consultivo, desenho de estudo, preparação de manuscrito, análise estatística, etc).

\section{REFERÊNCIAS}

1. Brasil. Ministério da Saúde. Secretaria de Atenção a Saúde. Departamento de Ações Programáticas Estratégicas. Portaria 1.994. Brasília. DF. 2009.

2. Brasil. Ministério da Saúde. Secretaria de Atenção a Saúde. Departamento de Ações Programáticas Estratégicas. Política Nacional de Atenção Integral à Saúde do Homem. Brasília. DF. 2008.

3. Korin D. Novas perspectivas de gênero em saúde. Adolescência Latinoamericana, Porto Alegre. $2001 ; 2(2): 67-79$.

4. Gomes R, Nascimento EF, Araújo FC. Por que os homens buscam menos os serviços de saúde do que as mulheres? As explicações de homens com baixa escolaridade e homens com ensino superior. Cad. Saúde Pública. 2007;23(3):565574. doi: $10.1590 /$ S0102-311X2007000300015

5. Figueiredo WS. Assistência à saúde dos homens: um desafio para os serviços de atenção primária. Ciência e Saúde Coletiva. 2005;10(1):105-109. doi: 10.1590/S1413$\underline{8123200500010001}$

6. Figueiredo WS, Schraiber LB. Concepções de gênero de homens usuários e profissionais de saúde de serviços de atenção primária e os possíveis impactos na saúde da população masculina, São Paulo, Brasil, Ciência e Saúde Coletiva. 2011 ; 16(supl.1):935-944. doi: 10.1590/S1413$\underline{81232011000700025}$

7. RIPSA - Rede Interagencial de Informações para a Saúde. Indicadores e Dados Básicos - IDB, Brasil, 2008.

8. Bahia. Plano Diretor de Regionalização. Secretaria da Saúde do Estado da Bahia, 2007.

9. Bahia. Secretaria da Saúde do Estado da Bahia. Regiões de Saúde do Estado da Bahia: 2007 a 2013, 2014 [Internet]. 2014 [Acessado em 06 agosto 2015]. Disponível em: http://www l.saude.ba.gov.br/mapa_bahia/docs/PDR _POP2012 _ atualizado10-07-2013.pdf 
10. Bittencourt SA, Camacho LAB, Leal MC. O Sistema de informação hospitalar e sua aplicação na saúde coletiva. Cad Saude Publica;2006;22(1):19-30.

11. Schraiber LB, Gomes R, Couto MT. Homens e saúde na pauta da Saúde Coletiva. Ciência \& Saúde Coletiva. 2005;10(1):7-17. doi: 10.1590/S141381232005000100002

12. Sabo D. $O$ estudo crítico das masculinidades. In. Adelman $M$, Silvestrin $C B$, organizadoras. Coletânea gênero plural. Curitiba: Editora UFPR; 2002. p. 33-46.

13. Laurenti R. Perfil epidemiológico da saúde masculina na Região das Américas. Uma contribuição para o enfoque de gênero. Faculdade de Saúde Pública/USP, São Paulo; 1998.

14. Bahia. Secretaria da Saúde do Estado da Bahia. Plano Estadual de Saúde: 2016 a 2019. Disponível em: http:// file:///C:/REC/Plano\%20Estadual\%20de $\% 20$ Saúde $\% 20$ \%202016-2019\%20VCES.pdf. Acesso em: 15 ago. 2017.

15. Mendes JDV, Sala A. Perfil da morbidade das internações masculinas no Estado de São Paulo. Bepa. 2011 ;8(93):22-31.

16. Oliveira NLB, Sousa RMC. Factors associated with the death of motorcyclists in traffic accident. Rev Esc Enferm USP. $2012 ; 46(6): 1380-7$.

17. Bastos YGL, Andrade SM, Soares DA. Características dos acidentes de trânsito e das vítimas atendidas em serviço pré-hospitalar em cidade do Sul do Brasil, 1997/2000. Cad Saúde Pública. 2005;21(3):815-22. doi: 10.1590/S0102$311 \times 2005000300015$

18. Rocha GS, Schor N. [Motorcycle accidents in the municipality of Rio Branco in the State of Acre: characterization and trends]. Ciên Saúde Coletiva. 2013;18(3):721-31.

19. Santos AMR, Batista MEM, Nunes BMVT, Leal CFS, Teles JBM. Perfil das vítimas de trauma por acidente de moto atendidas em um serviço público de emergência. Cad Saúde Pública. 2008;24(8):1927-38. doi: 10.1590/S0102$311 \times 2008000800021$

20. Lin MR, Kraus JF. A review of risk factors and patterns of motorcycle injuries. Accid Anal Prev. 2009;44(supl.4):710722, 2009. doi: 10.1016/i.aap.2009.03.010

21. Calil AM, Sallum EA, Domingues CA, Nogueira LS. Mapping injuries in traffic accident victims: a literature review. Rev Latino-Am Enfermagem. 2009;17(1):1 20-125. doi: 10.1590/S0104-11692009000100019

22. Barros AJD, Amaral RL, Oliveira MSB, Lima SC, Gonçalves EV. Motor vehicle accidents resulting in injuries: underreporting, characteristics, and case fatality rate. Cad Saúde Pública. 2003;19(4):979-86.
23. Andrade SM, Mello-Jorge MHP. Victims' characteristics by road accidents in a city of Southern Brazil. Rev Saúde Pública. 2000;34(2):149-56

24. Giles MF, Rothwell PM. Measuring the prevalence of stroke. Neuroepidemiology. 2008;30(4):205-6. doi: $10.1159 / 000126913$

25. Pereira $A B C N G$, Alvarenga $H$, Pereira Júnior RS, Barbosa MTS. Prevalência de acidente vascular cerebral em idosos no Município de Vassouras, Rio de Janeiro, Brasil, através do rastreamento de dados do Programa Saúde da Família. Revista Caderno Saúde Pública. 2009;25(9):1929-1936. doi: $10.1590 /$ S0102-311X2009000900007

26. Brasil. Ministério da Saúde. AVC: governo alerta para principal causa de mortes [Internet]. 2012 [Acesso em 02 junho de 2017]. Disponível em: http://www.brasil.gov.br/ saude/2012/04/acidente-vascular-cerebral-avc

27. Vieira EC, Cardoso ACC, Macêdo LB, Dias CMCC. Ocorrência de internações hospitalares por doenças do aparelho circulatório no estado da Bahia. Revista Pesquisa em Fisioterapia. 2016;6(2):1 15-123. doi: $10.17267 / 2238$ 2704rpf.v6i2.920

28. Laurenti, R, Mello-Jorge MHP, Gotlieb SLA. Perfil epidemiológico da morbi-mortalidade masculina. Ciência \& Saúde Coletiva. 2005;10(1):35-46. doi: 10.1590/S141381232005000100010

29. Toyoshima MTK, Ito GM, Gouveia N. Morbidade por doenças respiratórias em $\mathrm{p}$ acientes hospitalizados em São Paulo / SP. Rev Assoc Med Bras. 2005;51 (4):209-13. doi: $10.1590 /$ S0104-42302005000400017

30. Desalu OO, Oluwafemi JA, Ojo O. Morbidade e mortalidade relacionadas a doenças respiratórias em adultos atendidos em um hospital terciário na Nigéria. J Bras Pneumol. 2009;35(8):745-752. doi: 10.1590/S180637132009000800005

31. Brasil. Ministério da Saúde. Secretaria de Atenção à Saúde. Coordenação Geral de Saúde Mental. Reforma psiquiátrica e política de saúde mental no Brasil. Documento apresentado à Conferência Regional de Reforma dos Serviços de Saúde Mental: 15 anos depois de Caracas. OPAS. Brasília. DF. 2005. 